\title{
What to do after cardiac rehabilitation programs: the role of the general practitioner in cardiovascular prevention
}

\author{
Margaret Cupples, Neil Heron \\ Department of General Practice, UKCRC Centre of Excellence for Public Health Research, \\ Queen's University Belfast, Northern Ireland, UK
}

\begin{abstract}
This paper will review the current role of general practitioners (GPs) in providing cardiovascular prevention for patients after attendance at a cardiac rehabilitation program. Long-term implementation of preventive strategies is needed for continued impact on reducing risk of cardiovascular events and GPs have a major role in providing ongoing continuing medical care. Awareness of patients' social, cultural and physical circumstances allows the GP to identify individuals' needs for support in engaging in secondary prevention: relevant brief interventions can promote behaviour change in physical activity, diet and smoking habits, as well as promoting mental health and adherence to optimal medical therapy. Collaborative multidisciplinary working with community and hospital services provides best opportunities for timely referral to specialist expertise to maximise patients' well-being.
\end{abstract}

\section{Cardiac rehabilitation and the general practitioner}

General practitioners (GPs), together with other healthcare professionals working within primary care, have an important role in encouraging patients to maintain and further develop the health gains derived from cardiac rehabilitation (CR) programs. GPs carry responsibility for ensuring that, in the longer-term, after a cardiovascular diagnosis or acute event, medication for secondary prevention is prescribed appropriately and that cardiovascular risk factors are monitored and

Corresponding author: Prof. Margaret Cupples, Department of General Practice, UKCRC Centre of Excellence for Public Health Research, Queen's University Belfast University Road, Belfast, BT7 1NN, Northern Ireland, UK. Tel. +44.28.90245133. E-mail: M.Cupples@qub.ac.uk

Key words: Secondary prevention; cardiovascular disease; general practitioner; primary care; lifestyle change; risk factor monitoring.

Received for publication: 19 April 2016

Accepted for publication: 30 June 2016

CCopyright M. Cupples and Neil Heron, 2016

Tipografia PI-ME Editrice, Italy

Monaldi Archives for Chest Disease Cardiac Series 2016; 86:755

doi: 10.4081/monaldi.2016.755

This article is distributed under the terms of the Creative Commons Attribution Noncommercial License (by-nc 4.0) which permits any noncommercial use, distribution, and reproduction in any medium, provided the original author(s) and source are credited. minimised. Also, GPs are frequently involved in consultations concerning the early diagnosis and ongoing management of other illnesses and are often aware of particular circumstances existing within family and social networks. Thus, they are ideally placed to encourage ongoing adherence to secondary prevention following CR and to promote healthy lifestyle behaviour in a way that is relevant to the individual, their social setting and their cultural environment.

A health professional's communication of their perception of the importance of CR to patients is an important factor in encouraging attendance $[1,2]$ and their endorsement of an invitation to CR increases its likelihood of uptake [2,3]. Furthermore, patients appreciate having expert and practical advice provided by a trusted physician [4]. GPs will have knowledge of their patients' participation or non-participation in CR programs following a new cardiac diagnosis, event or intervention, so that they have an important role in advocating the value of CR and maintenance of implementation of its core components [5].

It is appropriate for the GP to explore reasons why patients may not participate in CR and to determine how a program may be made available, accessible and acceptable to them [6,7]. Some may not have been invited to participate in a program; for others, hospital and group based sessions may not be accessible or acceptable because of barriers such as lack of transport, inconvenient timing, social responsibilities or personal embarrassment [7]. For these, the GP may identify suitable home-based CR programs [8,9] or relevant community based initiatives that are based on principles of comprehensive CR and promote secondary prevention [10].

\section{General practice and cardiovascular disease prevention}

The GP has a role in both secondary and primary prevention of cardiovascular disease and should have clearly established links with the preventive services which may be based in hospital or the community, for their optimal delivery and avoidance of unnecessary duplication. They should ensure that patients understand advice given to them within CR programs and identify ongoing support for implementation of that advice and the achievement and maintenance of healthy lifestyles.

Health professionals who themselves have healthier lifestyle habits are more likely to counsel their patients about their lifestyles and to feel more confident in doing so [11]. Evidence indicates that those who are more physically active are more likely to educate their patients about physical activity and to motivate their patients to become more physically active [12]. Patients' perceptions of their GP's health status influences their perceptions of advice given about healthy eating and exercise: they often feel more confident about advice received from a GP whom they perceive as being healthy and are more likely to put that advice into practice [13]. 
Modifiable risk factors for cardiovascular diseases include not only physical inactivity and dietary factors but also smoking, excessive alcohol, hypertension, dyslipidaemia, diabetes, and obesity. These are addressed within comprehensive CR programs [5]; GPs contribute to their continued modification in lifelong secondary prevention.

\section{Secondary prevention in general practice}

A structured approach to the provision of secondary prevention leads to improved processes of care, including prescription of appropriate medication, and improved outcomes, including risk factor control [14], all-cause mortality and recurrent myocardial infarction [15]. In general practice, the organisation of regular recall of patients with established cardiovascular disease to review their health status, risk factors and medication, and to provide lifestyle advice, is effective in reducing patients' symptoms and improving their biophysical risk factor control and their diet and physical activity habits [16,17].

A recent systematic review confirmed an approximate $20 \%$ reduction in all-cause mortality and $26 \%$ reduction in risk of cardiac-related death in patients with cardiovascular disease who received structured programs of secondary prevention in primary care or community settings, compared to usual care [18]. However, evidence of a 'ceiling effect' was noted, whereby the additional effect of a specific organisational intervention on improving risk factor management is diminished when usual care is of high quality and follows similar principles of structured review. This was postulated as an explanation of the failure of a more recent intervention, that provided general practices with tailored management plans, to show benefit in risk factor control, although it was associated with a significant reduction in hospital admissions [19]. Of note, the effectiveness of interventions to improve the delivery of secondary prevention in general practice tend to diminish after the intervention ends [18], highlighting the need to emphasise that GPs should maintain focused attention on the structure of their plans for providing this care.

Different healthcare systems may facilitate the organisation of secondary prevention in different ways but it is worthwhile noting that the measurement and good control of biophysical risk factors does not alone necessarily maximise patients' well-being and quality of life. A comparative study of two different healthcare systems found that whilst risk factor control was better in one, which incentivised annual reviews of patients with cardiovascular disease by GPs, the diet and physical activity habits and quality of life of patients in the other system, with unstructured care provision, were better [20]. GPs should have good knowledge of their patients' family and social circumstances, with awareness of barriers to their optimal reduction in cardiovascular risk. Using this knowledge should facilitate the implementation of comprehensive secondary prevention [5], with maximal well-being, for all their patients.

\section{Promoting healthy lifestyle behaviours}

Whilst time for consultation with individual patients in general practice is limited, and intensive counselling may not be possible or practical, there is evidence for the effectiveness of brief interventions in promoting lifestyle change. It is important that GPs identify the different aspects of individuals' lifestyles which may be associated with cardiovascular risk so that they deliver relevant brief advice and offer onward referral to other professionals within the primary care team, community based services or hospital, as appropriate.

\section{Smoking cessation}

Smoking cessation is a key goal during and after CR [5]. Brief advice given by a physician has a positive effect on smoking cessation, increasing an unassisted quit rate by $1 \%-3 \%$ [21]. The approach of '5 A's', asking patients about their smoking habit, advising of the benefits of stopping, assessing their motivation to stop, assisting them in stopping and arranging a follow-up appointment to review progress, is recommended [22]. Assistance can be provided on an individual or group basis, face-to-face or using electronic communication systems and with or without pharmacotherapy [23]. Pharmacotherapy could include nicotine replacement therapy (NRT), varenicline or bupropion, prescribed with planned and agreed review intervals. Patients should be assisted to set an appropriate 'stop date' and plan for what to do if they fail. Social support for stopping may also be found among friends and family or within community based services.

In addition to referring or 'signposting' patients to cessation support services, GPs have a role in being an advocate for 'no smoking' public legislation and promoting no-smoking policies within local communities [24]. When social contacts smoke and fail to appreciate the adverse impact of smoking on health, this places barriers to patients' success in stopping smoking [4]. A no-smoking 'norm' within the social network of their family and community will encourage the adoption and maintenance of non-smoking behaviour.

\section{Physical activity}

GPs should provide active encouragement for patients to achieve recommended daily levels of physical activity across all population subgroups [25]. Identifying patients who are physically inactive and providing brief advice with possible referral to community-based walking or cycling programs or exercise schemes may support patients' engagement in physical activity. The cost-effectiveness of exercise referral schemes is uncertain [26] but community based walking programs and individual pedometer-based programs in general practice can be low cost and effective, both for the general population [27] and those who have participated in CR [28].

Clear guidance has been given for professionals who are involved in advising patients with cardiovascular disease regarding appropriate levels of physical activity for their health benefit [5]. GPs have a role in ensuring that patients have access to easy to read fact sheets, such as are available on the internet, so that they understand what is meant by recommendations regarding activities of varying intensity [29]. However, behaviour change requires not only the necessary knowledge and psychological and physical capacity to perform the behaviour but also requires opportunities to make it possible within an individual's physical, social and cultural environment [30]. Thus, strategies which seek to motivate individuals to change behaviours should ensure that they provide access to information that is clearly understood and access to the best possible conditions to promote uptake of advice.

The likelihood of patients with cardiovascular disease maintaining or increasing the level of physical activity they achieved during CR programs is greater if they live within a society that encourages physical activity. Behaviour change is difficult and whilst people are supported in making positive changes by positive social influences, they are also adversely influenced by peers, family and friends who advocate and engage in unhealthy behaviours [4]. A systematic review of interventions to promote physical activity in socioeconomically disadvantaged communities [31] found that group-based interventions had a positive impact on physical activity levels for adults and that interventions which provided a mix of professional guidance and on-going practical support were most likely to be effective [32]. 
GPs have a role in advising patients, in supporting community based initiatives, in alerting their patients to their availability and in advocacy with local agencies to ensure that the design and implementation of these initiatives are tailored to local context and community needs, including those of patients with cardiovascular disease. They also have a responsibility to recognise that patients are more likely to feel confident about their advice and follow it if they themselves are perceived to be healthy and physically active $[12,13]$.

\section{Healthy eating}

Healthy eating advice relevant to both the primary and secondary prevention of cardiovascular disease is focused on the 'Mediterranean diet', with increased consumption of fish, fruit, raw and cooked vegetables and olive oil [33,34]. Clinicians should routinely advise their patients to continue to adopt this approach to nutrition after completion of their CR program, in order to achieve lifelong optimal disease prevention. The provision of simple advice such as this after myocardial infarction has been associated with an increased frequency of consumption of these foods and a protective effect: higher levels of consumption of these foods were associated with a substantial reduction in the risk of early death [35].

In some communities barriers to complying with this advice may be a lack of availability of fresh fruit or vegetables or a cultural context that promotes eating 'fast foods'. Again GPs have a role in trying to ensure that the health education messages that are disseminated within $\mathrm{CR}$, as well as via public health campaigns, are understood and relevant to the local community. The optimal provision of healthcare is dependent on the collaborative interaction of multiple sectors of society and GPs should endeavour to work with policymakers and community leaders to reduce barriers, such as lack of food availability, cost and cooking habits, making the 'healthier choice' the 'easier choice' [36]. The value of social support for behavioural change in diet is recognised [4]. Secondary prevention programs which have included patients' family members or friends in planning management of cardiovascular risk reduction have shown good results in respect of improved dietary habits [37].

\section{Mental health}

Psychological distress, anxiety and depression are common amongst patients with cardiovascular disease, including those who have just completed CR programs [38]. In the ongoing monitoring of these patients' progress it is important for GPs to be alert to the potential diagnosis of depression. Asking patients if they have been bothered by feeling 'down, depressed or hopeless' or by having 'little interest or pleasure in doing things' are useful screening questions [39] which can be further explored with relevant validated clinical questionnaires [40].

The diagnosis of coronary heart disease and, in turn, its treatments may evoke stress [41] and lead to depression and the development of posttraumatic stress disorder. The impact of these conditions on patients' partners is important, as partners have the potential to affect patients' responses to their diagnosis and management [42]. GPs are uniquely placed to be aware of medical and psycho-social problems affecting family members and may alleviate these, reducing stress for both patients and their partners, by giving opportunity for fears to be revealed and discussed and for questions to be asked and answered to allow clear understanding and reassurance.

Patients with cardiovascular disease and depression are at increased risk of adverse outcomes [38]. Identifying and treating depression can be a major challenge as patients often have difficulty communicating problems with their mental health to others. Management options in general practice include provision of general health advice, counselling, on an individual or group basis, with cognitive-behavioural therapy, and anti-depressant medication [38]. Including partners or 'significant others' in management plans can help to ensure effective social support to address concerns and motivate healthy lifestyle habits. If there is no response to initial therapies, then options include referral for advice from specialist secondary care providers.

\section{Sexual activity}

Patients are often concerned about resuming sexual relations following a vascular diagnosis or event but may be too embarrassed to voice these concerns to health professionals. GPs who are aware of their patients' social circumstances may be pro-active in raising the issue, may help identify reasons for problems, including side-effects of medication, and provide reassurance regarding the safety of resumption of sexual activity, in accordance with guidance [33,43]. If GPs do not feel comfortable discussing this topic, they should adopt a nonjudgemental approach and signpost patients to appropriate resources, such as family planning or sexual health clinics.

\section{Alcohol consumption}

In response to stress, such as that associated with a cardiac event or diagnosis, some patients' consumption of alcohol may increase, or may be maintained at higher levels than recommended, with increased risk of further cardiovascular events. In general practice these individuals may be identified by using a validated alcohol screening questionnaire, such as The Alcohol-use disorders identification test (AUDIT) [44], of which an abbreviated version is available ('AUDIT-C'). GPs can offer a brief intervention using the 'FRAMES' principles (Feedback, Responsibility, Advice, Menu, Empathy, Self-efficacy) [45]. These include discussion of potential harm caused by drinking, reasons for changing behaviour, barriers to change and practical strategies to help, for example, setting specific goals. Such discussion may prevent development of adverse consequences. Patients who require more intensive interventions for problematic alcohol consumption can be signposted to appropriate services, such as community addiction teams, for specialist input.

\section{Pharmacological management}

Guidelines for the management of stable coronary heart disease include guidance regarding appropriate drug prescription [46]. They provide information about the appropriate targets for control of risk factors and the prescription of anti-platelet therapy, statins, beta-blockers and anti-hypertensive medications, as well as nitrates and other drugs for the control of symptoms. Prescribing guidelines in respect of specific first preference drugs may vary nationally and with time but GPs have a responsibility to be aware of these and to ensure that best practice in prescribing is adhered to for each individual patient. For example, one large observational study of patients with acute myocardial infarction found that only about half were discharged with optimal medical therapy compared with the standards in guidelines [47] and there is evidence that prescribing of secondary prevention medication across many European countries is suboptimal [48]. The GP has an important role in checking that patients receive appropriate medication prescription after CR and that they are fully involved in decision-making about prescribed medicines.

Patients should be aware of why they are taking specific medication, how to take it, its proposed benefits, potential side-effects and relevant cautions to be exercised when taking it. These discussions 
can be supported by appropriate written information, giving patients time to read and ask questions, making further appointments as necessary to ensure clear understanding and reach a well-informed shared decision about taking it. It is estimated that one third to a half of all medicines prescribed for long-term cardiovascular conditions are not taken as prescribed [49]. GPs can promote medication adherence by recommending the use of 'drug diaries', ensuring that the drug regime is clear, offering simple solutions to side-effects, encouraging the use of using containers that aid compliance and suggesting selfmonitoring, for example, of blood pressure, to provide feedback regarding risk factor control.

\section{Collaborative working}

An understanding of barriers which adversely influence motivation to follow healthy lifestyle advice and engage in secondary prevention is important. GPs are well placed to identify how preventive strategies can best be tailored to their patients' needs and to facilitate collaborative working between multidisciplinary professionals based in the community, primary care and hospital. New methods of exchanging information electronically between those involved in a patient's care are being developed and many will depend on the GP's contribution to co-ordinated teamwork. Integrated primary and secondary preventive care, involving patients and their families [50], based on a model of nurse-led multidisciplinary preventive cardiology [37] has shown improved lifestyle habits and better control of risk factors in both patients and their partners. GPs have an important role to play in ensuring that the provision of secondary cardiovascular disease prevention for their patients is optimal and maintained in the long-term, after attendance at cardiac rehabilitation.

\section{References}

1. Cooper AF, Jackson G, Weinman J, et al. Factors associated with cardiac rehabilitation attendance: a systematic review of the literature. Clin Rehabil 2002;16:541-5.

2. McCorry NK, Corrigan M, Tully MA, et al. Perceptions of exercise among people who have not attended cardiac rehabilitation following myocardial infarction. J Health Psychol 2009;14:924-32.

3. Grace SL, Russell KL, Reid RD, et al. Effect of cardiac rehabilitation referral strategies on utilization rates: a prospective, controlled study. Arch Intern Med 2011;171:235-41.

4. Cole JA, Smith SM, Hart N, et al. Do practitioners and friends support patients with coronary heart disease in lifestyle change? a qualitative study. BMC Fam Pract 2013;14:126.

5. Piepoli MF, Corrà U, Adamopoulos S, et al. Secondary prevention in the clinical management of patients with cardiovascular diseases. Core components, standards and outcome measures for referral and delivery. Eur J Prev Cardiol 2014;21:664-81.

6. Wingham J, Dalal HM, Sweeney KG, et al. Listening to patients: choice in cardiac rehabilitation. Eur J Cardiovasc Nurs 2006;5:289-94.

7. Dalal HM, Doherty P. Cardiac Rehabilitation. BMJ 2015;351:h5000.

8. Taylor RS, Dalal H, Jolly K, et al. Home-based versus centrebased cardiac rehabilitation. Cochrane Database Syst Rev 2015;8:CD007130.

9. Dalal HM, Evans PH. Achieving national service framework standards for cardiac rehabilitation and secondary prevention. BMJ 2003;326:481-4.

10. Piepoli MF, Corrà U, Benzer W, et al. Cardiac Rehabilitation Section of the European Association of Cardiovascular Prevention and
Rehabilitation. Secondary prevention through cardiac rehabilitation: from knowledge to implementation. A position paper from the Cardiac Rehabilitation Section of the EACPR. Eur J Cardiovasc Prev Rehabil 2010;17:1-17.

11. Howe M, Leidel A, Krishnan SM, et al. Patient-related diet and exercise counseling: do providers' own lifestyle habits matter? Prev Cardiol 2010;13:180-5.

12. Lobelo F, Duperly J, Frank E. Physical activity habits of doctors and medical students influence their counselling practices. Br J Sports Med 2009;43:89-92.

13. Fraser S, Leveritt M, Ball L. Patients' perceptions of their general practitioner's health and weight influences their perceptions of nutrition and exercise advice received. J Prim Health Care 2013;5:301-7.

14. McAlister FA, Lawson FM, Teo KK, et al. Randomised trials of secondary prevention programmes in coronary heart disease: systematic review. BMJ 2001;323:957-62.

15. Clark AM, Hartling L, Vandermeer B, et al. Meta-analysis: secondary prevention programs for patients with coronary artery disease. Ann Intern Med 2005;143:659-72.

16. Cupples ME, McKnight A. Randomised controlled trial of health promotion in general practice for patients at high cardiovascular risk. BMJ 1994;309:993-96.

17. Campbell NC, Ritchie LD, Thain J, et al. Secondary prevention in coronary heart disease: a randomised trial of nurse led clinics in primary care. Heart 1998;80:447-52.

18. Murphy E, Vellinga A, Byrne M, et al. Primary care organisational interventions for secondary prevention of IHD: a systematic review and meta-analysis. Br J Gen Pract 2015; 65:e460-8.

19. Murphy AW, Cupples ME, Smith SM, et al. Effect of tailored practice and patient care plans on secondary prevention of heart disease in general practice: cluster randomised controlled trial. BMJ 2009;339:b4220.

20. Cupples ME, Byrne MC, Smith SM, et al. Secondary prevention of cardiovascular disease in different primary healthcare systems with and without pay-for-performance. Heart 2008;94:1594-600.

21. Stead LF, Buitrago D, Preciado N, et al. Physician advice for smoking cessation. Cochrane Database Syst Rev 2013:5: CD000165.

22. U.S. Department of Health \& Human Services. Agency for Healthcare Research and Quality. Accessed on: April 1,2016. Available from: http://www.ahrq.gov/professionals/clinicians-providers/guidelinesrecommendations/tobacco/5steps.html

23. National Institute for Health and Care Excellence (NICE). Smoking cessation: supporting people to stop smoking. NICE quality standard 43, 2013. Accessed on: April 1, 2016. Available from: https://www.nice.org.uk/guidance/qs43.

24. Shelley E, Cupples M. Health promotion for the general public. In: S. Gielen, G. De Backer, M. Piepoli and D. Wood (eds.), The ESC Textbook of Preventive Cardiology. Oxford University Press 2015;24:303-11.

25. Department of Health. UK physical activity guidelines, 2011. Accessed on: April 1, 2016. Available from: https://www.gov.uk/government/publications/uk-physical-activity-guidelines

26. Campbell F, Holmes M, Everson-Hock E, et al. A systematic review and economic evaluation of exercise referral schemes in primary care: a short report. Health Technol Assess 2015;19:60.

27. Heron N, Tully MA, McKinley MC, et al. Steps to a better Belfast: physical activity assessment and promotion in primary care. Br J Sports Med 2014;48:1558-63.

28. Cupples ME, Dean A, Tully MA, et al. Using pedometer step-count goals to promote physical activity in cardiac rehabilitation: a feasibility study of a controlled trial. Int J Phys Med Rehabil 2013;1:7. 
29. NHS Choices. Your health, your choices. Accessed on: April 1, 2016. Available from: http://www.nhs.uk/Livewell/fitness/Pages/physicalactivity-guidelines-for-adults.aspx

30. Michie S, Van Stralen MM, West R. The behaviour change wheel: a new method for characterising and designing behaviour change interventions. Implement Sci 2011;6:42.

31. Cleland CL, Tully MA, Kee F, et al. The effectiveness of physical activity interventions in socio-economically disadvantaged communities: A systematic review. Prev Med 2012;54:371-80.

32. Hillsdon M, Foster C, Thorogood M. Interventions for promoting physical activity. Cochrane Database Syst Rev 2005;1:CD003180

33. Perk J, De Backer G, Gohlke H, et al. European Guidelines on cardiovascular disease prevention in clinical practice. Eur Heart $\mathrm{J}$ 2012;33:1635-701.

34. Sofi F, Abbate R, Gensini GF, et al. Accruing evidence on benefits of adherence to the Mediterranean diet on health: an updated systematic review and meta-analysis. Am J Clin Nutr 2010; 92:1189-96.

35. Barzi F, Woodward M, Marfisi RM, et al. GISSI-Prevenzione Investigators. Mediterranean diet and all-causes mortality after myocardial infarction: results from the GISSI-Prevenzione trial. Eur J Clin Nutr 2003;57:604-11.

36. World Health Organization. Ottawa Charter for Health Promotion. First International Conference on Health Promotion, Ottawa, 21 November 1986. Available from: http//www.who.int/healthpromotion/ conferences/previous/ottawa/en

37. Wood DA, Kotseva K, Connolly S, et al. Nurse-coordinated multidisciplinary, family-based cardiovascular disease prevention programme (EUROACTION) for patients with coronary heart disease and asymptomatic individuals at high risk of cardiovascular disease: a paired, cluster-randomised controlled trial. Lancet 2008;371:1999-2012.

38. Pogosova N, Saner H, Pedersen SS, et al. Psychosocial aspects in cardiac rehabilitation: from theory to practice. A position paper from Cardiac Rehabilitation Section of the European Association of Cardiovascular Prevention and Rehabilitation of the European Society of Cardiology. Eur J Prev Cardiol 2015;22:1290-306.

39. National Institute for Health and Care Excellence (NICE). Depression in adults with a chronic physical health problem - Treatment and Management NICE clinical guideline 91, 2009. Available from: https://www.nice.org.uk/guidance/cg91
40. Kiely KM, Butterworth P. Validation of four measures of mental health against depression and generalized anxiety in a community based sample. Psychiatry Res 2015; 225:291-8.

41. von Känel R, Hari R, Schmid JP, et al. Distress related to myocardial infarction and cardiovascular outcome: a retrospective observational study. BMC Psychiatry 2011;11:98.

42. Moser DK, Dracup K. Role of spousal anxiety and depression in patients'psychosocial recovery after a cardiac event. Psychosom Med 2004;66:527-32.

43. Levine GN, Steinke EE, Bakaeen FG, et al. Sexual activity and cardiovascular disease: a scientific statement from the American Heart Association. Circulation 2012;125:1058-72.

44. Lundin A, Hallgren M, Balliu N, et al. The use of alcohol use disorders identification test (AUDIT) in detecting alcohol use disorder and risk drinking in the general population: validation of AUDIT using schedules for clinical assessment in neuropsychiatry. Alcohol Clin Exp Res 2015;39:158-65.

45. Health Education for England. Alcohol IBA - 04 Clinical approach to brief advice: structuring brief advice. Department of Health, London, 2009. Accessed on: April 1, 2016. Available from: http://www.alcohollearningcentre.org.uk/alcoholeLearning/learnin g/IBA/Module4_v2/D/ALC_Session/300/session.html

46. Taske Force Members, Montalescot G, Sechtem U, et al. 2013 ESC guidelines on the management of stable coronary artery disease: the Task Force on the management of stable coronary artery disease of the European Society of Cardiology. Eur Heart J 2013;34:2949-3003.

47. Bramlage P, Messer C, Bitterlich N, et al. The effect of optimal medical therapy on 1-year mortality after acute myocardial infarction. Heart 2010;96:604-09.

48. Kotseva K, Wood D, De Bacquer D, et al. EUROASPIRE IV: a European Society of Cardiology Survey on the lifestyle, risk factor and therapeutic management in coronary patients from 24 European countries. Eur J Prev Cardiol 2016;23:636-48.

49. National Institute for Health and Care Excellence (NICE). Medicines adherence: Involving patients in decisions about prescribed medicines and supporting adherence. NICE clinical guideline 76, 2009. Available from: https://www.nice.org.uk/guidance/cg76

50. Connolly S, Holden A, Turner E, et al. MyAction: an innovative approach to the prevention of cardiovascular disease in the community. Br J Car 2011;18:171-6. 